\title{
The difference between fasting and non-fasting lipid measurements is not related to statin treatment
}

\author{
Ming-Ming Liu\#, Jia Peng", Ye-Xuan Cao, Yuan-Lin Guo, Na-Qiong Wu, Cheng-Gang Zhu, Ying Gao, \\ Jian-Jun Li
}

State Key Laboratory of Cardiovascular Diseases, Fu Wai Hospital, National Center for Cardiovascular Diseases, Chinese Academy of Medical Sciences and Peking Union Medical College, Beijing, China

Contributions: (I) Conception and design: JJ Li, Y Gao, MM Liu; (II) Administrative support: JJ Li; (III) Provision of study materials or patients: Y Gao, YL Guo, NQ Wu, CG Zhu; (IV) Collection and assembly of data: MM Liu, J Peng, YX Cao; (V) Data analysis and interpretation: MM Liu, J Peng; (VI) Manuscript writing: All authors; (VII) Final approval of manuscript: All authors.

\#These authors contributed equally to this work.

Correspondence to: Jian-Jun Li, MD, PhD, FESC, FACC; Ying Gao, MD. State Key Laboratory of Cardiovascular Diseases, Fu Wai Hospital, National Center for Cardiovascular Diseases, Chinese Academy of Medical Sciences and Peking Union Medical College, No. 167 BeiLiShi Road, Xicheng District, Beijing 100037, China. Email: lijianjun938@126.com; cmuxyg@sina.com.

\begin{abstract}
Background: Non-fasting blood samples are routinely used to assess plasma lipid profiles except in patients with severe hypertriglyceridemia according to the previous consensus. However, the impact of statin use on non-fasting plasma lipid measurements has not been thoroughly evaluated.

Methods: In this cross-sectional study, 686 patients with normal triglyceride (TG) levels, who were hospitalized due to chest pain, were enrolled. Fasting (8-12 h) and non-fasting (2-4 h after breakfast) lipid profiles were measured on the second day of admission. Patients were divided into the non-statin $(n=499)$ group and statin treatment $(\mathrm{n}=187)$ group. Differences in fasting and non-fasting lipid profiles between the statin and non-statin groups were compared.

Results: The mean age of participants was $57 \pm 13$ years, and $54.4 \%$ were male. A linear correlation was observed between fasting and non-fasting lipid profiles. Although a postprandial impact on lipid parameters was observed, the general pattern of differences between fasting and non-fasting lipids was similar in both groups. Additionally, the $\operatorname{diff}(\%)$ [(non-fasting-fasting)/fasting $\times 100 \%]$ of lipid panels did not vary by statin treatment. Moreover, no effects of statin types or duration of use on non-fasting lipid profiles were identified.

Conclusions: The current study found fasting and non-fasting lipid panels were similar in individuals with or without statin treatment. Non-fasting lipid panels were not significantly affected by statin types or duration of use, suggesting that non-fasting lipid measurement is an acceptable test for patients receiving statin treatment.
\end{abstract}

Keywords: Non-fasting; statin; triglyceride (TG); cholesterol

Submitted May 15, 2020. Accepted for publication Nov 02, 2020.

doi: $10.21037 / \mathrm{atm}-20-3962$

View this article at: http://dx.doi.org/10.21037/atm-20-3962

\section{Introduction}

People mainly live in the non-fasting state during a regular 24-hour cycle. Therefore, non-fasting lipid panels may be a reliable indicator of average lipid concentration level because the fasting state only occurs after fasting for at least 8 hours (1). The advantage of non-fasting over fasting lipid measurements is a simplified blood collection process for patients and physicians that is suitable for children, the elderly, and diabetic patients. For convenience, Denmark's health services laboratories recommended implementation of a random non-fasting profile test in 2009 (2). Numerous clinical trials have shown that non-fasting low-density 
lipoprotein cholesterol (LDL-C) has prognostic value that is similar to fasting levels. Non-fasting triglyceride (TG) levels are associated with an increased risk of coronary heart disease (CHD), similar to the increased risk associated with fasting concentrations (3). This observation has led to the notion that non-fasting lipids may be an equivalent or better predictor of cardiovascular conditions since non-fasting lipid profiles can reflect real status of circulating lipids (4).

Non-fasting lipid measurements are not "universally applicable", and may require additional fasting lipid testing under certain clinical conditions. First, an additional laboratory test is required if non-fasting samples are initially analyzed for glucose or therapeutic drug monitoring. Second, the European consensus has recommended that laboratories offer re-measurement of fasting TGs if non-fasting TG levels are $\geq 350 \mathrm{mg} / \mathrm{dL}$, because TG concentrations are more stable in the fasting state (1). Additionally, patients recovering from hypertriglyceridemia-induced pancreatitis or with diagnosed hypertriglyceridemia ( $\mathrm{TG} \geq 200 \mathrm{mg} / \mathrm{dL}$ according to American Heart Association guidelines) should be assessed fasting lipids during clinic follow-up (5). Finally, medications that can cause hypertriglyceridemia may affect non-fasting lipid profiles, thus emphasizing the need for repeated fasting measurements.

The impact of lipid-lowering medications on non-fasting lipid profiles has not been thoroughly evaluated. Statins, the most widely used lipid-lowering drugs, are known to reduce LDL-C by up to $50 \%$ and decrease TGs by $20 \%$ (6). Although non-fasting samples have been collected in several clinical trials following statin therapies (7-9), little information is currently available regarding differences between fasting and non-fasting lipid measurements in statintreated individuals (10). In this study, we aimed to evaluate whether non-fasting lipid testing is clinically acceptable in patients receiving statin therapy and to examine the impact of statin types and duration of use on non-fasting lipid tests. We present the following article in accordance with the STROBE reporting checklist (available at http://dx.doi. org/10.21037/atm-20-3962).

\section{Methods}

\section{Study population}

The study cohort was prospectively designed to examine the difference between fasting and non-fasting lipid profiles. The study complied with the Declaration of Helsinki (as revised in 2013) and was approved by the hospital's ethical review board (Fu Wai Hospital \& National Center for Cardiovascular Diseases, Beijing, China, approval number: 2013-442). Informed written consents were obtained from all patients enrolled in this study.

From April 2015 to October 2018, 1,280 patients, scheduled for coronary angiography due to angina-like chest pain, were recruited at Fuwai Hospital (Figure 1). Patients with complete baseline demographic data and lipid tests (including fasting and non-fasting blood samples) were selected for this study. Sixty-one patients were excluded due to missing non-fasting blood samples, 458 patients were excluded because they had known hypertriglyceridemia ( $\mathrm{TG} \geq 200 \mathrm{mg} / \mathrm{dL}$ ), and 47 patients were excluded due to missing values, severe hepatic or renal insufficiency, thyroid dysfunction, systemic inflammatory disease, or malignant tumor. Patients receiving other lipid-lowering therapies, or lower-intensity or high-intensity statin therapy before admission $(n=28)$ were also excluded. Finally, 686 patients were enrolled in the final analysis and assigned to either moderate-intensity statin therapy before admission $(n=187$; statin group) or without statin therapy before admission ( $\mathrm{n}=499$; non-statin group).

Fasting blood samples were collected on the second day of admission following an 8-12-hour period of fasting; non-fasting blood samples were collected on the same day 2-4 hours after breakfast $(1,11)$. High-intensity statin therapy was defined as equivalent to atorvastatin $40 / 80 \mathrm{mg} / \mathrm{d}$ or rosuvastatin $20 / 40 \mathrm{mg} / \mathrm{d}$. Moderate-intensity statin therapy was defined as equivalent to atorvastatin $10 / 20 \mathrm{mg} / \mathrm{d}$, rosuvastatin $5 / 10 \mathrm{mg} / \mathrm{d}$, simvastatin $20 / 40 \mathrm{mg} / \mathrm{d}$, pravastatin $40 / 80 \mathrm{mg} / \mathrm{d}$ or pitavastatin $2 / 4 \mathrm{mg} / \mathrm{d}$. Low-intensity statin therapy was defined as simvastatin $10 \mathrm{mg} / \mathrm{d}$, pravastatin $10 / 20 \mathrm{mg} / \mathrm{d}$ or pitavastatin $1 \mathrm{mg} / \mathrm{d}$ (5). Hypertriglyceridemia was defined as a TG concentration $\geq 200 \mathrm{mg} / \mathrm{dL}$, according to American or Chinese panels (12).

Fasting and non-fasting lipid profiles, including total cholesterol (TC), TG, LDL-C, high-density lipoprotein cholesterol (HDL-C), remnant cholesterol, and non-HDL-C, were analyzed in all participants. Remnant cholesterol was calculated as TC minus LDL-C minus HDL-C, which was the cholesterol content of all triglyceride-rich lipoproteins, including chylomicron remnants, very-low-density lipoprotein (VLDL), and intermediate-density lipoproteins (IDL) in the fasting or non-fasting states (13). Non-HDL-C was calculated as TC minus HDL-C, equivalent to the combined LDL-C, remnant cholesterol, and lipoprotein(a) cholesterol. Calculated LDL-C was defined by Friedewald equation as follows: LDL-C $(\mathrm{mg} / \mathrm{dL})=\mathrm{TC}(\mathrm{mg} / \mathrm{dL})-$ 


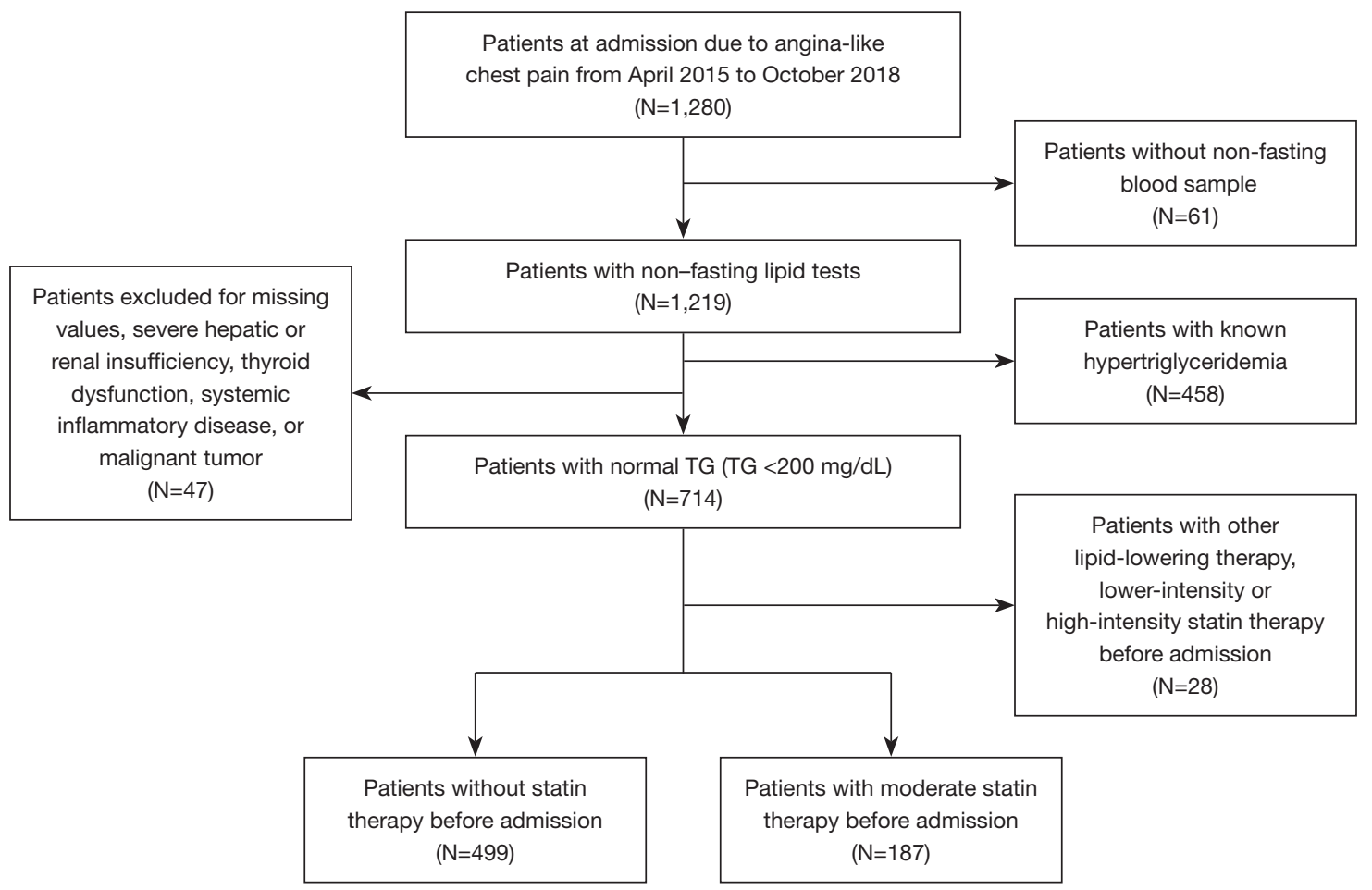

Figure 1 Flowchart of patient enrollment.

HDL-C (mg/dL) - TG (mg/dL)/5. The $\operatorname{diff}(\%) s$ of lipid panels were calculated as follows: (non-fasting - fasting)/ fasting $\times 100 \%$.

Hypertension was defined as systolic blood pressure $\geq 140 \mathrm{mmHg}$ or diastolic blood pressure $\geq 90 \mathrm{mmHg}$, and/or currently taking antihypertensive drugs, and/or self-reported history of hypertension (14). Diabetes mellitus was defined by fasting plasma glucose $\geq 7.0 \mathrm{mmol} / \mathrm{L}$, the 2 -h plasma glucose of the oral glucose tolerance test $\geq 11.1 \mathrm{mmol} / \mathrm{L}$, glycated hemoglobin $\geq 6.5 \%$, and/or current use of hypoglycemic drugs or insulin, and/or self-reported history of diabetes (15). Body mass index (BMI) was calculated by height and weight (16).

\section{Laboratory examination}

Blood samples were collected through venipuncture and analyzed by clinical laboratory. Lipid profiles were measured using an automatic biochemistry analyzer (Hitachi 7150, Tokyo, Japan) by an enzymatic assay (17). In detail, TC was measured by the cholesterol oxidase-p-aminophenazone method (CHOD-PAP, Cholesterol kit, BioSino Biotechnology \& Science Inc., Beijing, China). TG was measured by the glycerine phosphate oxidase peroxidase method (GPOPAP, Triglyceride kit, BioSino Biotechnology \& Science
Inc., Beijing, China). LDL-C was analyzed by the selective solubilization method (Low Density Lipid Cholesterol Test Kit, Kyowa Medex, Tokyo, Japan). HDL-C was determined by a similar method (Determiner L HDL, Kyowa Medex, Tokyo, Japan). $\mathrm{Lp}$ (a) was assayed by an immunoturbidimetry method according to the manufacturer's guide as previously described [LASAY Lp(a) auto; SHIMA Laboratories, Tokyo, Japan] with a normal value of $<30 \mathrm{mg} / \mathrm{dL}$ (18). HbA1c was measured using a Tosoh Automated Glycohemoglobin Analyzer (HLC-723G8, Tokyo, Japan).

\section{Statistical analysis}

Continuous variables are shown as mean \pm standard deviation (SD) or median [interquartile range (IQR)]. Categorical variables are expressed as number (percentage). Differences between groups were analyzed using Student's $t$-tests and $\chi^{2}$ tests. Spearman rank correlation coefficients (r) were used to assess correlations between fasting and non-fasting lipid profiles. Fasting and non-fasting lipid profiles were compared using a nonparametric test of two paired samples (Wilcoxon signed-rank test). The diff(\%)s in individuals with or without statins were compared by the Mann-Whitney $\mu$ test. The Bland-Altman analysis was conducted to assess agreement 
between fasting and non-fasting lipid tests. Statistical analyses were performed using SPSS software (version 25, SPSS, Chicago, Illinois, USA). All tests were two-sided and defined statistical significance by $\mathrm{P}<0.05$.

\section{Results}

\section{Baseline characteristics}

The final dataset included 686 patients, with 187 assigned to the statin group and 499 assigned to the non-statin group. Baseline characteristics of participants in both groups are summarized in Table 1. The mean age of participants was $57 \pm 13$ years, and $54.4 \%$ were male. There were no significant differences between the two groups in baseline variables, history of diabetes, or alcohol consumption. Notably, the percentage of participants with coronary artery disease (CAD) and hypertension was higher in the statin group. Patients with statin therapy were prescribed more aspirin and angiotensin-converting enzyme inhibitor (ACEI) in the hospital. In addition, the proportion of laboratory tests was similar in both groups.

\section{Changes and correlations between fasting and non-fasting lipids}

There were significant differences between fasting and nonfasting levels for all lipid parameters $(\mathrm{P}<0.001)$. As shown in Table 2, a slight increase in the median (IQR) levels of TGs and remnant cholesterol was observed from the fasting to the non-fasting state. In contrast, a minor decrease in the median concentrations of TC, HDL-C, LDL-C and nonHDL-C was found from the fasting to the non-fasting state.

The absolute change in postprandial lipid levels was $+0.19 \mathrm{mmol} / \mathrm{L}$ for TG, $-0.05 \mathrm{mmol} / \mathrm{L}$ for TC, $-0.02 \mathrm{mmol} / \mathrm{L}$ for HDL-C, $-0.10 \mathrm{mmol} / \mathrm{L}$ for direct LDL-C, $-0.13 \mathrm{mmol} / \mathrm{L}$ for calculated LDL-C, $+0.06 \mathrm{mmol} / \mathrm{L}$ for remnant cholesterol and $-0.03 \mathrm{mmol} / \mathrm{L}$ for non-HDL-C. The Bland-Altman analysis showed that the difference between fasting and nonfasting levels was within the total acceptable error allowed for each lipid parameter (Figure 2). Moreover, fasting lipid levels correlated well with non-fasting lipid levels, and further examination showed similar a linear correlation (Figure 3).

\section{Comparison between fasting and non-fasting lipids in statin and non-statin groups}

Mean differences between fasting and non-fasting lipid concentrations were similar in patients with and without statin treatment (Table 2). There were no significant differences for all lipid parameters between the statin and non-statin groups. In addition, non-HDL-C levels were not influenced by food intake in the statin-treated group, but median concentrations levels decreased by -0.05 ( -0.30 to $0.21) \mathrm{mmol} / \mathrm{L}$ in the non-statin group.

Furthermore, the diff(\%)s [(non-fasting - fasting)/fasting $\times 100 \%$ ] for all lipid variables were not statistically different between the statin and non-statin groups. Diff(\%)s of TG were $16.5 \%$ in the statin-treated and $16.1 \%$ in the nonstatin group. Similarly, the $\operatorname{diff}(\%)$ s were $-0.6 \%$ and $-1.1 \%$ for TC, $-3.9 \%$ and $-2.1 \%$ for HDL-C, $-4.8 \%$ and $-2.9 \%$ for direct LDL-C, $-5.4 \%$ and $-5.3 \%$ for calculated LDL-C, and $14.8 \%$ and $12.0 \%$ for remnant cholesterol in the statin and non-statin groups, respectively. Additionally, the diff(\%)s for non-HDL-C were modestly different between the statin $(0.0 \%)$ and the non-statin groups $(-1.5 \%)$.

\section{Comparison between fasting and non-fasting lipids in statin types, and duration of use}

Among all participants in the statin-treated group, 132 patients were prescribed moderate-intensity atorvastatin (ATO) or rosuvastatin (ROS), while 55 patients were prescribed other types of statins. Median difference values and $\operatorname{diff}(\%)$ s for all lipid parameters were not significantly different between patients prescribed ATO or ROS and those prescribed other types of statins (Table 3). Similar results were found in individuals prescribed moderateintensity statin therapy for 3 to 12 months and individuals with statin treatment for at least 1 year. The diffs(\%) for each lipid parameter were not statistically different when comparing short-term $(<1$ year) with long-term $(>1$ year) statin therapy (Table 4). Therefore, fasting and non-fasting lipids were not affected by statin types or duration of use.

\section{Discussion}

In this cross-sectional study, we found that the general pattern of differences between fasting and non-fasting lipid levels were similar in statin and non-statin groups. Diffs(\%) for all lipid parameters were not statistically significant in both groups regardless of statin types or duration of use. These findings suggest that non-fasting lipid measurements may be reliable for patients currently receiving statin therapy who do not have hypertriglyceridemia.

Previous studies, including the Copenhagen General 
Table 1 Baseline characteristics of individuals in statin and non-statin group

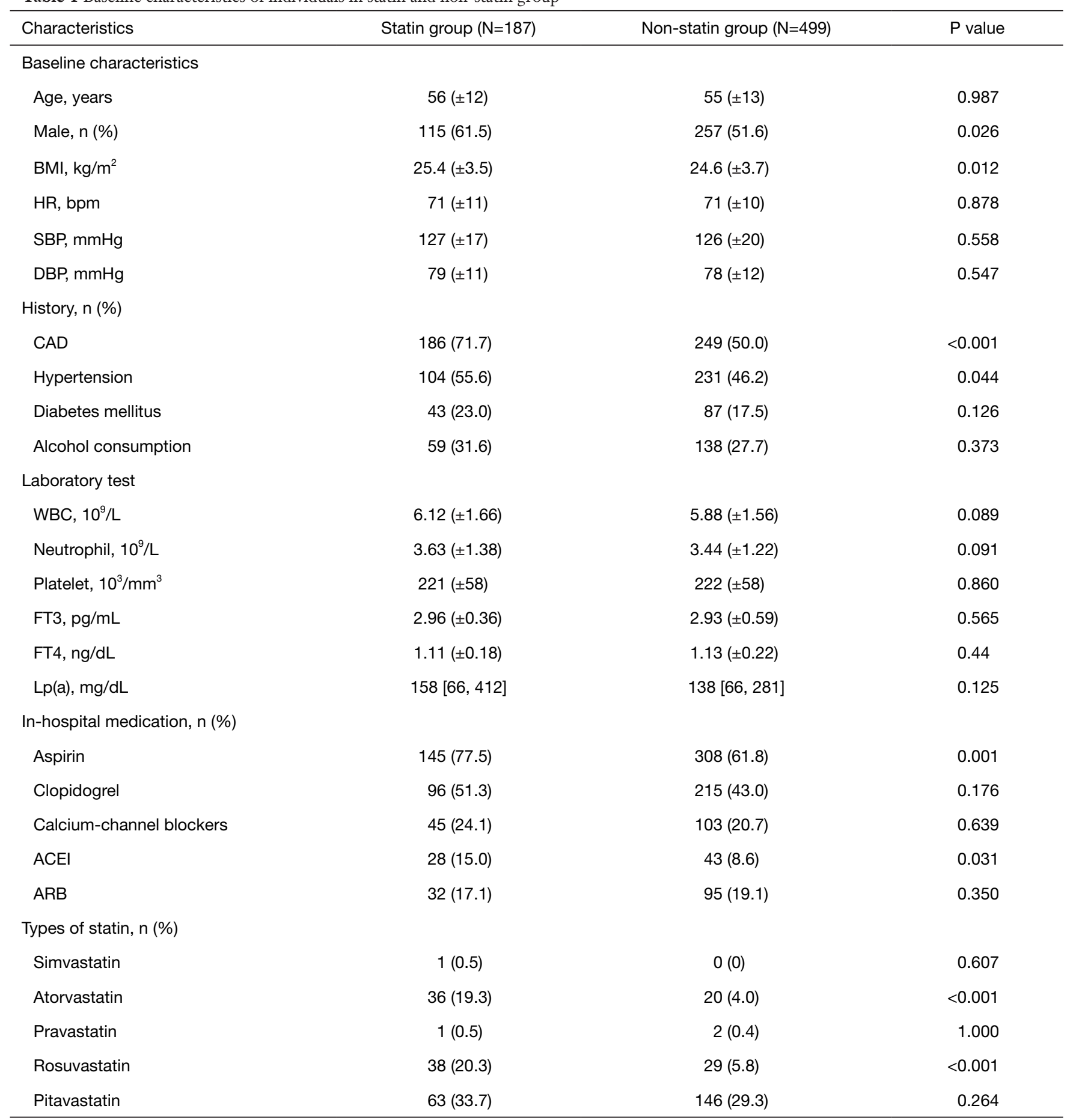

Data are mean $( \pm \mathrm{SD})$, median [interquartile range] and number (\%) of patients. BMI, body mass index; HR, heart rate; SBP, systolic blood pressure; DBP, diastolic blood pressure; CAD, coronary artery disease; WBC, white blood cell; FT3, free triiodothyronine; FT4, free thyroxine; Lp(a), lipoprotein(a); ACEI, angiotensin converting enzyme inhibitor; ARB, angiotensin receptor blockers. 
Table 2 Comparison between fasting and non-fasting lipid profiles stratified by statin and non-statin group

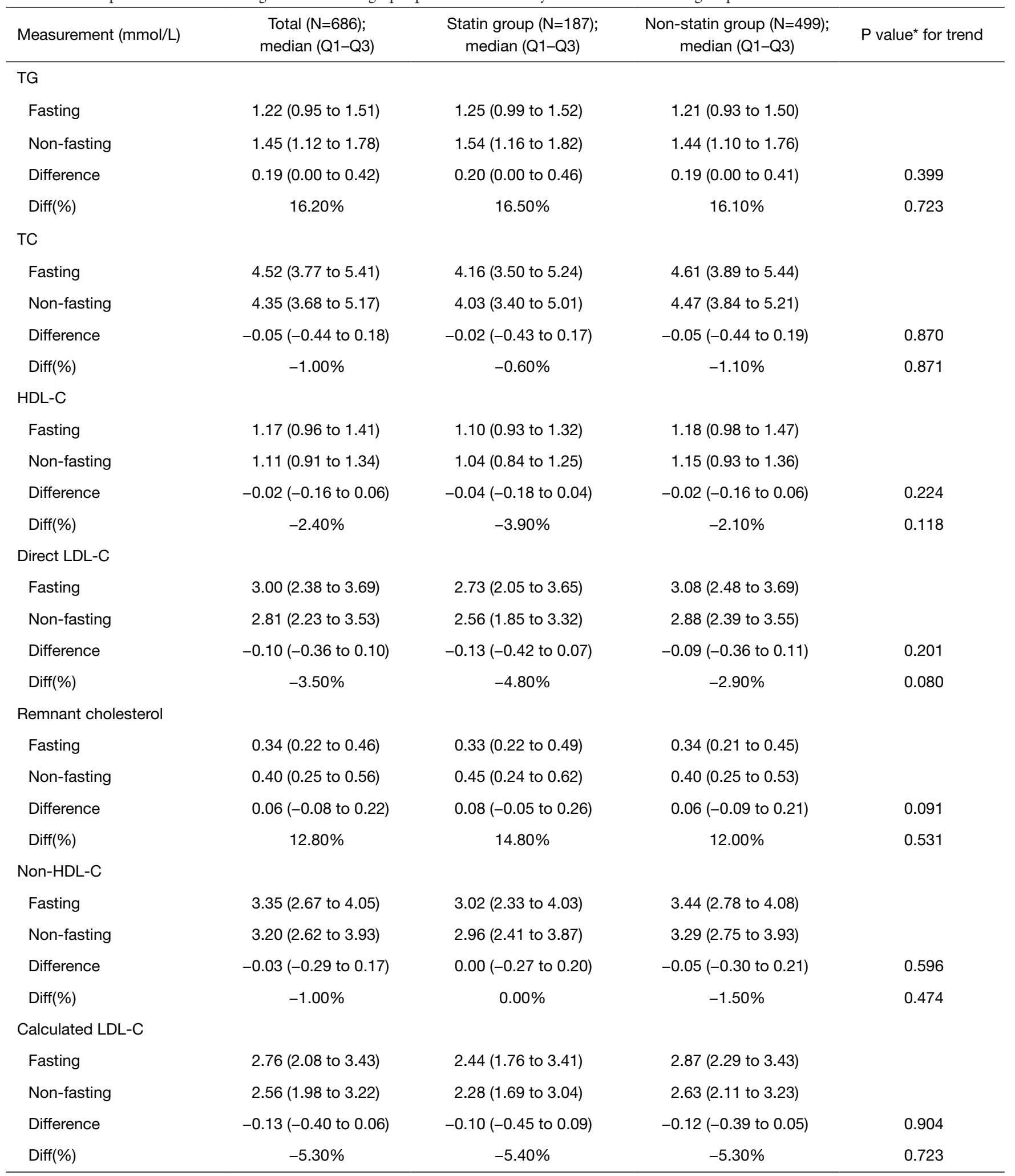

*, $\mathrm{P}<0.05$ suggests significant difference. $\mathrm{P}$ value for difference between statin and non-statin group and diff(\%) is calculated by MannWhitney U test. TG, triglyceride; TC, total cholesterol; HDL-C, high-density lipoprotein cholesterol; LDL-C, low-density lipoprotein cholesterol. 
A

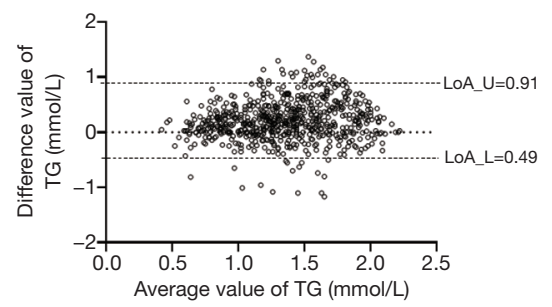

D

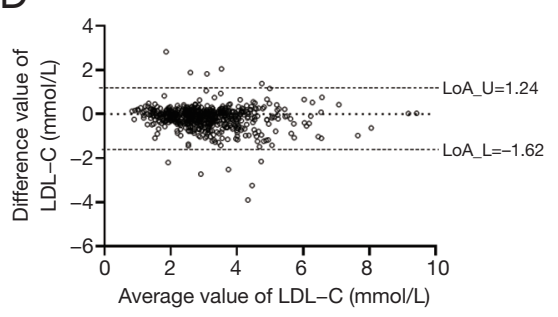

B

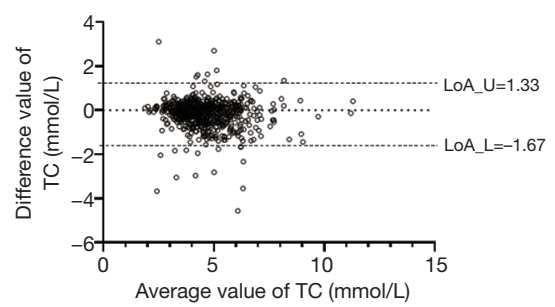

E

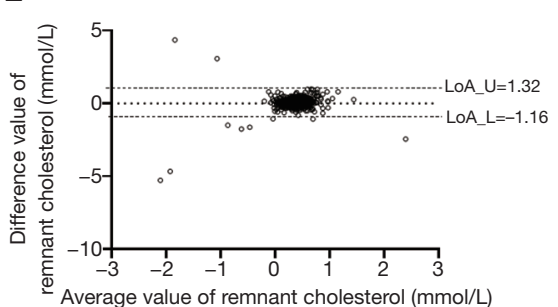

C

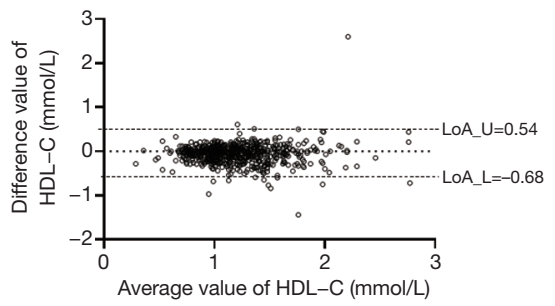

$\mathrm{F}$

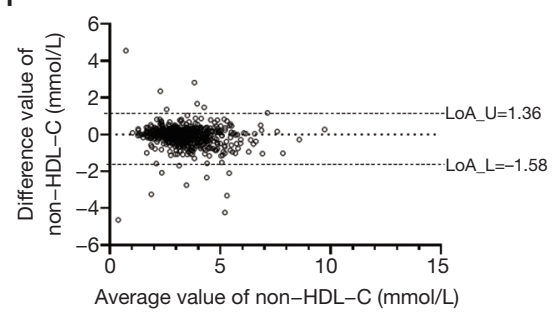

Figure 2 The Bland-Atlman plot of each lipid parameters. The Bland-Altman plot displays the mean difference and limits of agreement (LoA). Confidential intervals for LoA are shown as hidden line. TG, triglyceride; TC, total cholesterol; HDL-C, high-density lipoprotein cholesterol; LDL-C, low-density lipoprotein cholesterol; U, upper limit; L, lower limit.
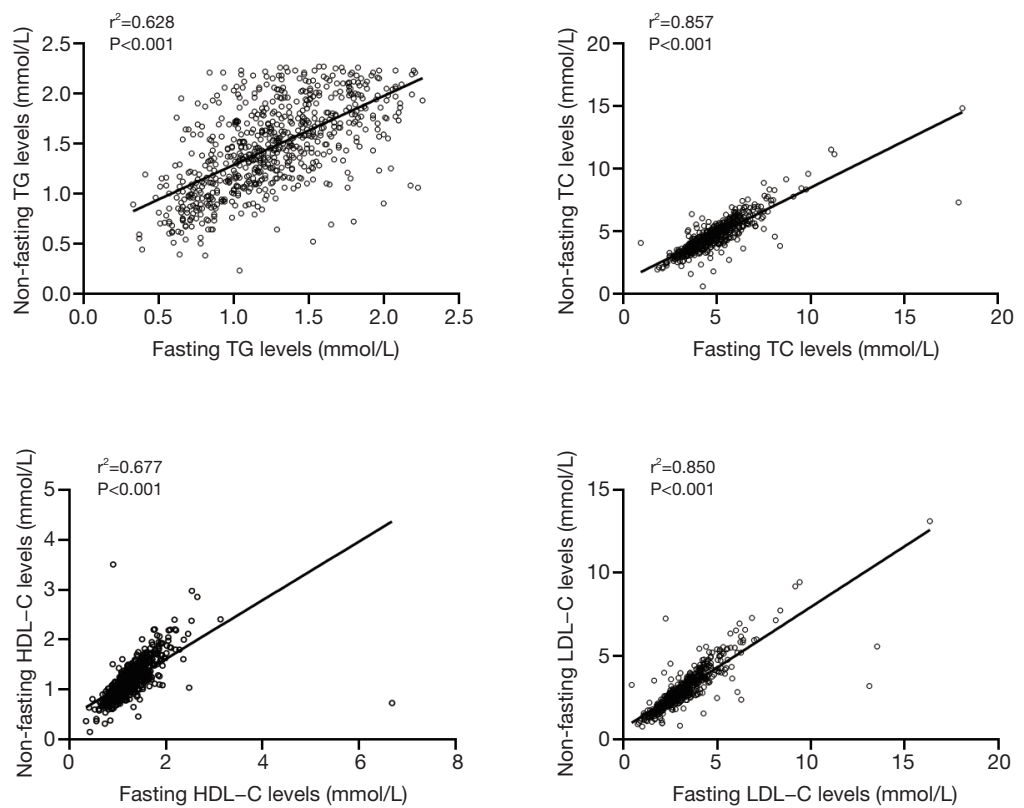

Figure 3 Correlations between fasting and non-fasting lipids ( $\mathrm{N}=686$ ). TG, triglyceride; TC, total cholesterol; HDL-C, high-density lipoprotein cholesterol; LDL-C, low-density lipoprotein cholesterol.

Population Study, the Women's Health Study in the USA, the National Health and Nutrition Examination Survey in the USA, and Calgary Laboratory Services in Canada, have examined the utility of non-fasting lipid testing to define standard lipid parameters. Their results indicated that non-fasting lipid profiles changed minimally in response 
Table 3 Comparison between fasting and non-fasting lipid profiles stratified by types of statin

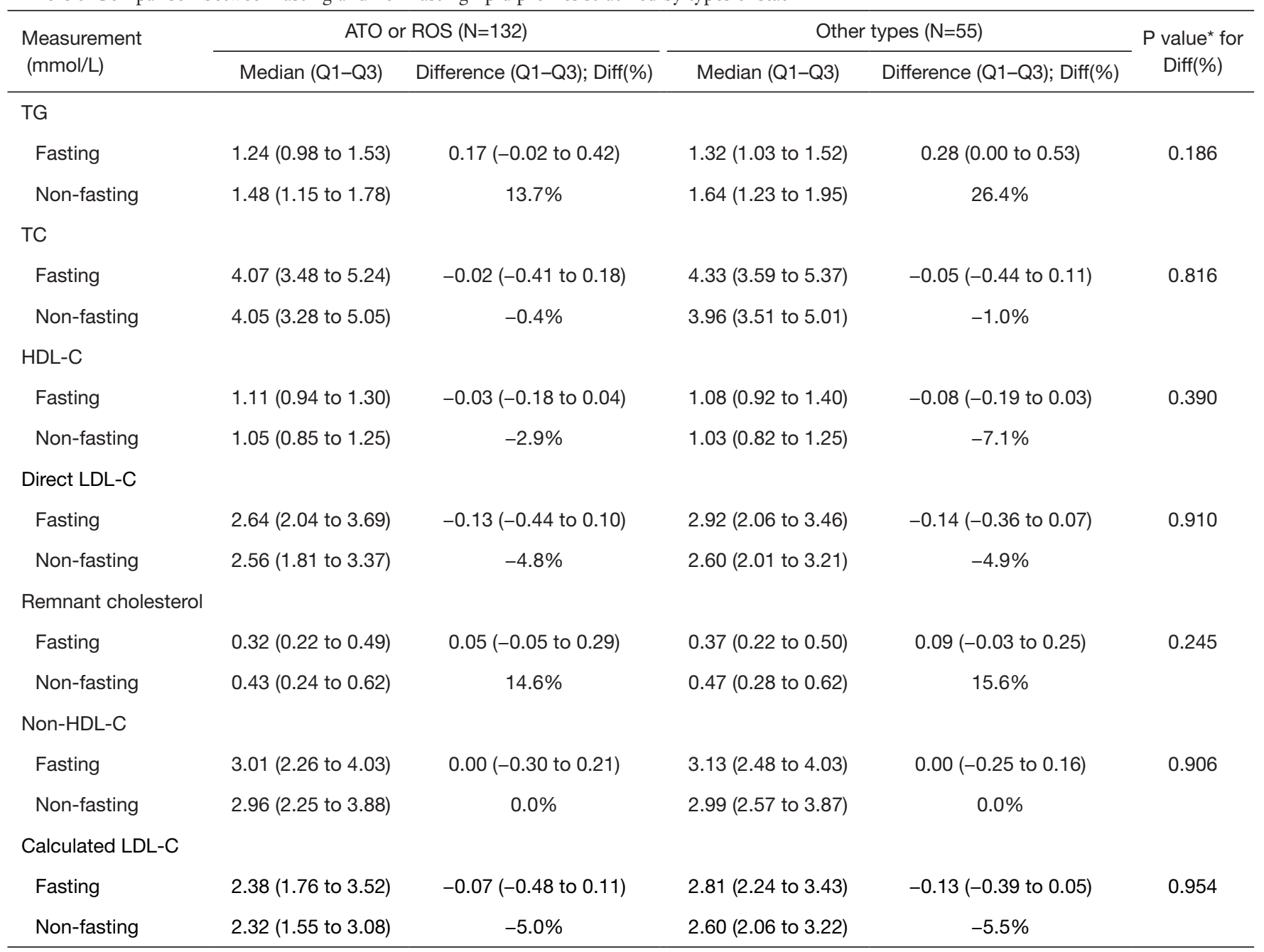

*, $\mathrm{P}<0.05$ suggests significant difference. $\mathrm{P}$ value for diff(\%) is calculated by Mann-Whitney $\mathrm{U}$ test between different types of statin subgroups. ATO, atorvastatin; ROS, rosuvastatin; TG, triglyceride; TC, total cholesterol; HDL-C, high-density lipoprotein cholesterol; LDL-C, low-density lipoprotein cholesterol.

to regular food intake $(3,19-21)$. Average postprandial changes were $+0.3 \mathrm{mmol} / \mathrm{L}$ for $\mathrm{TG},-0.2 \mathrm{mmol} / \mathrm{L}$ for $\mathrm{TC}$, $-0.1 \mathrm{mmol} / \mathrm{L}$ for HDL-C, and $-0.2 \mathrm{mmol} / \mathrm{L}$ for LDL-C. Although the extent of changes for these lipid parameters in our study differed slightly from the average values reported above, the trend we observed were identical to the findings of these large clinical trials. It is worth mentioning that non-HDL-C levels were similar in the fasting and non-fasting state in the statin group during the study. Theoretically, non-HDL-C is equivalent to the combined LDL-C and remnant cholesterol (22). Although LDL-C decreased and remnant cholesterol increased in the nonfasting state, non-HDL-C levels remained unchanged. The reason behind this observation could be that non-HDL-C levels were calculated by TC and HDL-C levels, and both parameters decreased negligibly after breakfast in the statin group (23).

Fasting lipid panels have been the standard in clinical practice for the past half-century since the fasting TG levels were recommended in the 1967 classification of hyperlipidemia (24). Since then, fasting lipids have been widely used in real-world clinical practice to reduce measurement variability for TG and TC. Although fasting lipids may lead to a more accurate Friedewald-calculated LDL-C, the inconvenience of fasting lipid measurement has decreased patient compliance for lipid analysis. Recent 
Table 4 Comparison between fasting and non-fasting lipid profiles stratified by durations of statin treatment

\begin{tabular}{|c|c|c|c|c|c|}
\hline $\begin{array}{l}\text { Measurement } \\
(\mathrm{mmol} / \mathrm{L})\end{array}$ & \multicolumn{2}{|c|}{ Statin therapy $<1$ year $(\mathrm{N}=165)$} & \multicolumn{2}{|c|}{ Statin therapy $\geq 1$ year $(\mathrm{N}=22)$} & $\begin{array}{l}\text { P value* for } \\
\text { Diff(\%) }\end{array}$ \\
\hline \multicolumn{6}{|l|}{ TG } \\
\hline Fasting & 1.21 (0.94 to 1.49$)$ & 0.21 (0.00 to 0.46$)$ & 1.53 (1.27 to 1.70$)$ & $0.12(-0.04$ to 0.37$)$ & 0.205 \\
\hline Non-fasting & 1.46 (1.12 to 1.82$)$ & $17.4 \%$ & 1.70 (1.58 to 1.91$)$ & $7.2 \%$ & \\
\hline Fasting & 4.13 (3.50 to 5.23$)$ & $-0.04(-0.40$ to 0.17$)$ & 4.56 (3.20 to 5.90$)$ & $-0.01(-0.98$ to 0.27$)$ & 0.500 \\
\hline Non-fasting & 4.03 (3.48 to 5.03$)$ & $-0.7 \%$ & 4.01 (3.19 to 4.77$)$ & $-0.3 \%$ & \\
\hline \multicolumn{6}{|l|}{ HDL-C } \\
\hline Fasting & $1.13(0.95$ to 1.34$)$ & $-0.04(-0.18$ to 0.04$)$ & $0.92(0.78$ to 1.17$)$ & $-0.04(-0.25$ to 0.05$)$ & 0.496 \\
\hline Fasting & 2.68 (2.05 to 3.59$)$ & $-0.13(-0.38$ to 0.07$)$ & 3.13 (1.99 to 4.32$)$ & $-0.15(-0.98$ to 0.18$)$ & 0.427 \\
\hline Non-fasting & 2.58 (1.91 to 3.31$)$ & $-4.7 \%$ & 2.39 (1.70 to 3.40$)$ & $-5.9 \%$ & \\
\hline \multicolumn{6}{|c|}{ Remnant cholesterol } \\
\hline Fasting & $0.32(0.22$ to 0.49$)$ & $0.08(-0.04$ to 0.26$)$ & $0.43(0.24$ to 0.57$)$ & $0.05(-0.11$ to 0.38$)$ & 0.642 \\
\hline Non-fasting & $0.45(0.24$ to 0.60$)$ & $14.8 \%$ & $0.48(0.35$ to 0.64$)$ & $14.1 \%$ & \\
\hline \multicolumn{6}{|l|}{ Non-HDL-C } \\
\hline Fasting & 3.01 (2.33 to 3.93$)$ & $0.00(-0.25$ to 0.21$)$ & 3.49 (2.22 to 4.90$)$ & $0.00(-0.82$ to 0.21$)$ & 0.320 \\
\hline Non-fasting & 2.96 (2.42 to 3.89$)$ & $0.0 \%$ & 2.93 (2.14 to 3.82 ) & $0.0 \%$ & \\
\hline
\end{tabular}

*, $\mathrm{P}<0.05$ suggests significant difference. $\mathrm{P}$ value for diff(\%) is calculated by Mann-Whitney $\mathrm{U}$ test between short-term and long-term statin subgroups. TG, triglyceride; TC, total cholesterol; HDL-C, high-density lipoprotein cholesterol; LDL-C, low-density lipoprotein cholesterol.

studies have demonstrated the reliability of non-fasting TG for predicting risk of myocardial infarction, ischemic heart disease, and cardiovascular death, suggesting that non-fasting lipids may be useful for cardiovascular risk prediction (25). A meta-analysis examining 68 prospective studies reported that non-fasting blood samples did not attenuate the value for cardiovascular risk prediction (26). Therefore, based on the present evidence, non-fasting lipids may be similar, or superior, to fasting lipids for cardiovascular risk predictions.

Whether non-fasting lipid tests are suitable for the patients currently receiving statin therapy has not been resolved prior to our study. The utility of non-fasting lipids has been examined in at least three statin-related clinical trials, including the Heart Protection Study, the lipid-lowering arm of the Anglo-Scandinavian Cardiac Outcomes Trial (ASCOT-LLA), and the Study of the Effectiveness of Additional Reductions in Cholesterol and Homocysteine (SEARCH) (7-9). Unfortunately, these studies did not focus on the impact of statin therapy on non-fasting lipids. Previous large randomized clinical trials allocated statins to participants in a fixed manner, including type and dose, making it impossible to observe the effects of different statins on non-fasting lipid levels. Given the limitations of previous clinical trials mentioned above, we saw the opportunity to study the potential impact of statin treatment, including statin types and administration 
duration, on non-fasting lipid tests.

The current study design may have several advantages compared to previous studies regarding the impacts of statin use on non-fasting lipid tests. First, the fasting and non-fasting blood samples were assayed on the same day. In addition, the effects of different statins and administration duration on the lipid profile of non-fasting lipids were examined. Moreover, patients in the statin group received moderate-intensity statin therapy for at least 3 months before hospitalization without other types of lipid-lowering drugs, such as ezetimibe or fibrate. Finally, the study excluded patients with hypertriglyceridemia in advance to strictly control the main confounding factor, TG, in the measurement of non-fasting LDL-C. Hence, the present study might provide additional, novel information regarding the clinical implication of nonfasting lipid tests in real world practice.

Nevertheless, there were several limitations of the present study. First, sample sizes were relatively small, which might have contributed to the borderline significance for some analyses (Table 2). Hence, additional investigations with larger sample sizes are needed in the future. Second, a single-center study may be another limitation, as ethnic differences and different lifestyles may lead to different results between Western and Chinese populations. Since our research enrolled only the Chinese participants, the results need to be confirmed in other racial groups.

\section{Conclusions}

The current study on Chinese participants showed a general pattern of differences between fasting and non-fasting lipid profiles in the statin and non-statin groups. At the same time, the data also indicated that the differences between fasting and non-fasting lipid measurements were not affected by statin types or duration of use. These findings are novel and suggest that non-fasting lipid tests are clinically acceptable for patients receiving statin treatment.

\section{Acknowledgments}

Funding: This study was partly supported by Capital Health Development Fund (201614035) and Chinese Academy of Medical Sciences Innovation Fund for Medical Sciences (2016-I2M-1-011) awarded to Dr. Jian-Jun Li, MD, PhD.

\section{Footnote}

Reporting Checklist: The authors present the study in accordance with the STROBE reporting checklist. Available at http://dx.doi.org/10.21037/atm-20-3962

Data Sharing Statement: Available at http://dx.doi. org/10.21037/atm-20-3962

Peer Review File: Available at http://dx.doi.org/10.21037/ atm-20-3962

Conflicts of Interest: All authors have completed the ICMJE uniform disclosure form (available at: http://dx.doi. org/10.21037/atm-20-3962). The authors have no conflicts of interest to declare.

Ethical Statement: The authors are accountable for all aspects of the work in ensuring that questions related to the accuracy or integrity of any part of the work are appropriately investigated and resolved. The study complied with the Declaration of Helsinki (as revised in 2013) and was approved by the hospital's ethical review board (Fu Wai Hospital \& National Center for Cardiovascular Diseases, Beijing, China, approval number: 2013-442). Informed written consents were obtained from all patients enrolled in this study.

Open Access Statement: This is an Open Access article distributed in accordance with the Creative Commons Attribution-NonCommercial-NoDerivs 4.0 International License (CC BY-NC-ND 4.0), which permits the noncommercial replication and distribution of the article with the strict proviso that no changes or edits are made and the original work is properly cited (including links to both the formal publication through the relevant DOI and the license). See: https://creativecommons.org/licenses/by-nc-nd/4.0/.

\section{References}

1. Nordestgaard BG, Langsted A, Mora S, et al. Fasting Is Not Routinely Required for Determination of a Lipid Profile: Clinical and Laboratory Implications Including Flagging at Desirable Concentration Cutpoints-A Joint Consensus Statement from the European Atherosclerosis Society and European Federation of Clinical Chemistry and Laboratory Medicine. Clin Chem 2016;62:930-46.

2. Nordestgaard BG, Hilsted L, Stender S. Plasma lipids in non-fasting patients and signal values of laboratory results. Ugeskr Laeger 2009;171:1093.

3. Langsted A, Nordestgaard BG. Nonfasting versus fasting lipid profile for cardiovascular risk prediction. Pathology 
2019;51:131-41.

4. Kolovou GD, Anagnostopoulou KK, Daskalopoulou SS, et al. Clinical relevance of postprandial lipaemia. Curr Med Chem 2005;12:1931-45.

5. Arnett DK, Blumenthal RS, Albert MA, et al. 2019 ACC/AHA Guideline on the Primary Prevention of Cardiovascular Disease: A Report of the American College of Cardiology/American Heart Association Task Force on Clinical Practice Guidelines. Circulation 2019;140:e596-e646.

6. Awad K, Serban MC, Penson P, et al. Effects of morning vs evening statin administration on lipid profile: A systematic review and meta-analysis. J Clin Lipidol 2017;11:972-985.e9.

7. MRC/BHF Heart Protection Study of cholesterollowering therapy and of antioxidant vitamin supplementation in a wide range of patients at increased risk of coronary heart disease death: early safety and efficacy experience. Eur Heart J 1999;20:725-41.

8. Mora S, Chang CL, Moorthy MV, et al. Association of Nonfasting vs Fasting Lipid Levels With Risk of Major Coronary Events in the Anglo-Scandinavian Cardiac Outcomes Trial-Lipid Lowering Arm. JAMA Intern Med 2019;179:898-905.

9. Armitage J, Bowman L, Wallendszus K, et al. Intensive lowering of LDL cholesterol with $80 \mathrm{mg}$ versus 20 $\mathrm{mg}$ simvastatin daily in 12,064 survivors of myocardial infarction: a double-blind randomised trial. Lancet 2010;376:1658-69.

10. Abdel-Aziza W, Soltana G, Amer AA. Comparison between fasting and nonfasting lipid profile in patients receiving treatment with statin therapy. Menoufia Medical Journal 2017;30:614.

11. Simundic AM, Cornes M, Grankvist K, et al. Standardization of collection requirements for fasting samples: for the Working Group on Preanalytical Phase (WG-PA) of the European Federation of Clinical Chemistry and Laboratory Medicine (EFLM). Clin Chim Acta 2014;432:33-7.

12. Jellinger PS, Handelsman Y, Rosenblit PD, et al. American Association of Clinical Endocrinologists and American College of Endocrinology Guidelines for management of dyslipidemia and prevention of cardiovascular disease executive summary. Endocr Pract 2017;23:479-97.

13. Nordestgaard BG, Varbo A. Triglycerides and cardiovascular disease. Lancet 2014;384:626-35.

14. Williams B, Mancia G, Spiering W, et al. 2018 ESC/ESH Guidelines for the management of arterial hypertension: The Task Force for the management of arterial hypertension of the European Society of Cardiology and the European Society of Hypertension: The Task Force for the management of arterial hypertension of the European Society of Cardiology and the European Society of Hypertension. J Hypertens 2018;36:1953-2041.

15. American Diabetes Association. 1. Improving Care and Promoting Health in Populations: Standards of Medical Care in Diabetes-2019. Diabetes Care 2019;42:S7-S12.

16. Menke A, Casagrande S, Geiss L, et al. Prevalence of and Trends in Diabetes Among Adults in the United States, 1988-2012. JAMA 2015;314:1021-9.

17. Cao YX, Liu HH, Sun D, et al. The different relations of PCSK9 and Lp(a) to the presence and severity of atherosclerotic lesions in patients with familial hypercholesterolemia. Atherosclerosis 2018;277:7-14.

18. Li S, Wu NQ, Zhu CG, et al. Significance of lipoprotein(a) levels in familial hypercholesterolemia and coronary artery disease. Atherosclerosis 2017;260:67-74.

19. Mora S, Rifai N, Buring JE, et al. Fasting compared with nonfasting lipids and apolipoproteins for predicting incident cardiovascular events. Circulation 2008;118:993-1001.

20. Steiner MJ, Skinner AC, Perrin EM. Fasting might not be necessary before lipid screening: a nationally representative cross-sectional study. Pediatrics 2011;128:463-70.

21. Sidhu D, Naugler C. Fasting time and lipid levels in a community-based population: a cross-sectional study. Arch Intern Med 2012;172:1707-10.

22. Nordestgaard BG. A Test in Context: Lipid Profile, Fasting Versus Nonfasting. J Am Coll Cardiol 2017;70:1637-46.

23. Iso H, Naito Y, Sato S, et al. Serum triglycerides and risk of coronary heart disease among Japanese men and women. Am J Epidemiol 2001;153:490-9.

24. Fredrickson DS, Levy RI, Lees RS. Fat transport in lipoproteins--an integrated approach to mechanisms and disorders. N Engl J Med 1967;276:34-42.

25. Nordestgaard BG, Benn M, Schnohr P, et al. Nonfasting triglycerides and risk of myocardial infarction, ischemic heart disease, and death in men and women. JAMA 2007;298:299-308.

26. Di Angelantonio E, Sarwar N, Perry P, et al. Major lipids, apolipoproteins, and risk of vascular disease. JAMA 2009;302:1993-2000.

Cite this article as: Liu MM, Peng J, Cao YX, Guo YL, Wu NQ, Zhu CG, Gao Y, Li JJ. The difference between fasting and non-fasting lipid measurements is not related to statin treatment. Ann Transl Med 2021;9(5):386. doi: 10.21037/atm20-3962 